\title{
Design of FIR Filters for Fast Multiscale Directional Filter Banks
}

\author{
Epiphany Jebamalar Leavline ${ }^{\# 1}$ and Shunmugam Sutha ${ }^{* 2}$ \\ \#Department of ECE, Bharathidasan Institute of Technology, Anna University, \\ Tiruchirappalli - 620 024, India \\ *Department of EEE, University College of Engineering, Dindigul-624 622, India \\ ${ }^{1}$ jebi.lee@gmail.com \\ ${ }^{2}$ suthapadmanabhan@gmail.com
}

\begin{abstract}
Fast multiscale directional filter bank $(F M D F B)$ is a multiscale image representation scheme that is suitable for several image processing applications such as image denoising, texture retrieval, and pattern recognition. It is constructed using the iterative filter structures namely Laplacian pyramid and directional filter bank. In this paper, we present the method for designing the FIR filters required to construct FMDFB. Also, we demonstrate the perfect reconstruction and critically sampled property of FMDFB.
\end{abstract}

Keywords: FMDFB, Filter design, Laplacian pyramid, Directional Filter Banks, Wavelet transform

\section{Introduction}

Wavelet transform is a mathematical tool that finds application in many fields of engineering and technology. Wavelet transform is good in detecting point discontinuities [1]. But the natural images have such point discontinuities arranged along smooth contours (e.g., edges). Wavelet cannot link these point discontinuities to represent a meaningful shape, thus it is not capable of representing edges that are high frequency components effectively [2].

In fact, wavelet transform decomposes the high frequency components only into three directional subbands (horizontal, vertical, and $45^{\circ}$ diagonal) [3]. One can see the edges in these high frequency subbands, yet the directional information of the edges is not seen from the high frequency subbands. Due to this issue, more number of wavelet coefficients at different scales is required to represent and to reconstruct the edges without loss [2]. This led to the evolution of an efficient multiscale image representation schemes that are local, directional and support multiresolution expansion. An efficient multiscale image representation scheme namely pyramidal directional filter banks or the contourlet transform [4]. This is an iterated filter bank structure that uses a pyramidal transform namely Laplacian pyramid (LP) for scale decomposition and a directional transform namely directional filter banks (DFB) for directional decomposition that links point discontinuities to a meaningful shape.

The MDFB alter the pyramidal directional filter bank or Contourlet Transform by introducing scale decomposition in its finest scale at additional computational complexity [5]. Cheng, et al., [6] proposed a fast structure for this MDFB named as fast multiscale directional filter banks (FMDFB). This filter structure is found suitable for various image processing applications such as image retrieval, feature extraction, and denoising. The FMDFB is constructed with two basic building blocks namely Laplacian pyramid (LP) and directional 
filter bank (DFB). Both LP and DFB are iterative filter structures that are realized using separable 1D FIR filters.

This paper presents the design procedure of FIR filters required for constructing the basic building blocks of FMDFB. The perfect reconstruction nature of the FMDFB is demonstrated with the experimental results. The rest of the paper is organized as follows. Section 2 describes the basic building blocks of FMDFB. In Section 3, the design of FIR filters for FMDFB is presented. The experimental results are discussed in Section 4 and the paper is concluded in Section 5.

\section{Fast Multiscale Directional Filter Banks}

Cheng et al proposed a fast and critically sampled structure for MDFB namely as fast multiscale directional filter banks [6] shown in Figure 1. The basic building blocks of Fast Multiscale Directional Filter banks are Laplacian pyramid (LP) and directional filter banks (DFB).
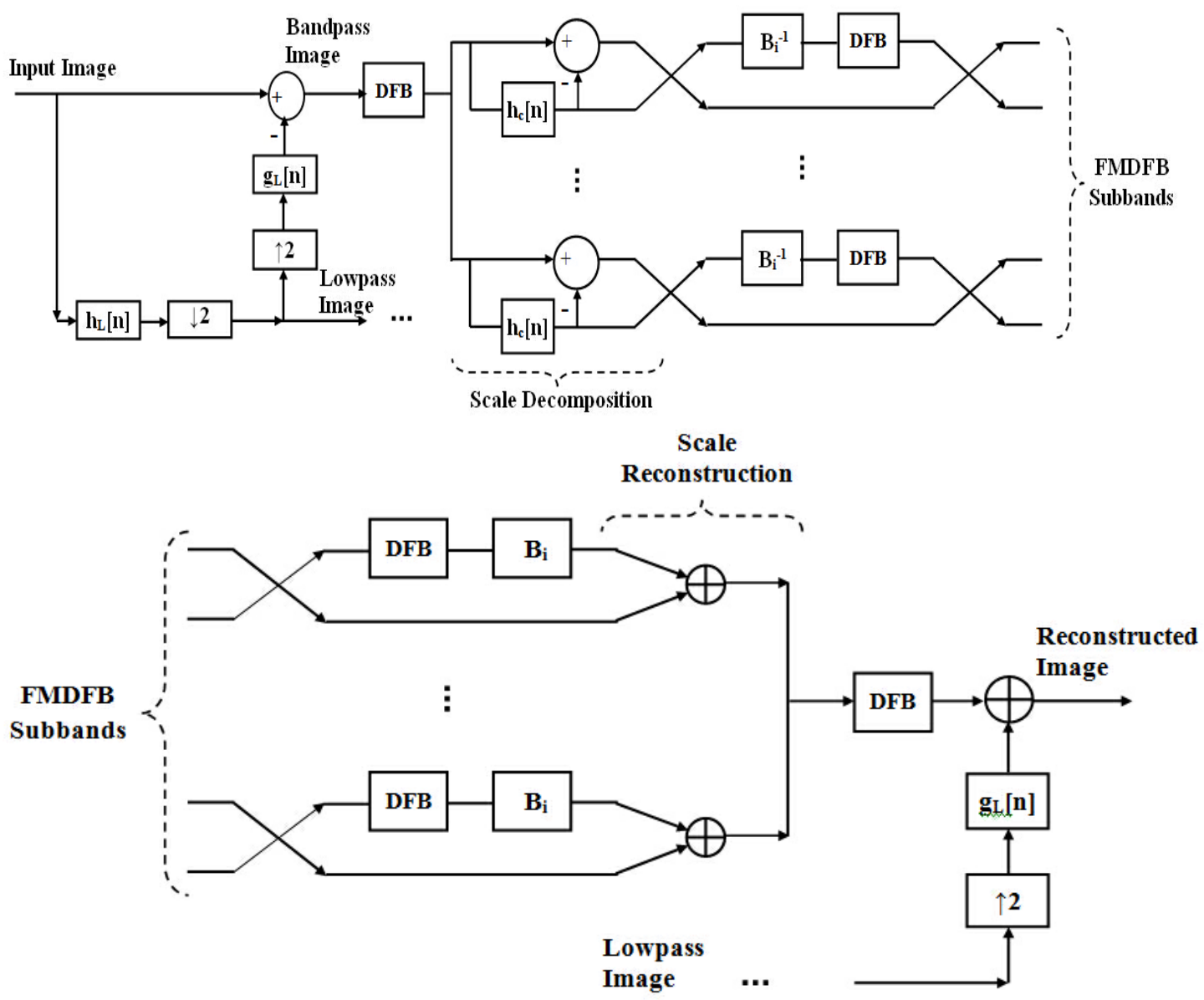

Figure 1. (a) Analysis Filter Bank Structure for FMDFB (b) Synthesis Filter Bank Structure for FMDFB. $h_{L}$ and $g_{L}$ are the Lowpass and Highpass Filters Respectively in LP. Bi and $\mathrm{Bi}^{-1}$ Represents the Back Sampling and Inverse Back Sampling. DFB is the Directional Filter Bank that Performs Directional Decomposition 
In analysis filter bank structure, low pass filtering and wavelet transform technique are applied for splitting the image into various scales. Use of non aliasing lowpass filters broadens the bandwidth of the finer scale. Directional decomposition with lower angular resolution is performed before scale decomposition. Hence, one set of operations for directional decomposition with lower angular resolution is saved by sharing. Figure 2 shows the frequency resolution of contourlet, MDFB and FMDFB. Also, the total number of subband coefficients will be equal to the size of the original image and thereby maintaining the maximally decimated property [7]. As stated in [6], perfect reconstruction is always possible at all scales regardless of the low-pass filters used for decomposition. According to Cheng et.al this FMDFB achieved 33.5\% - 37.5\% of reduction in computational complexity when compared to original MDFB.

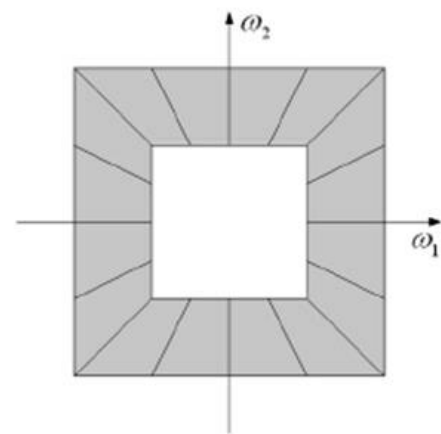

(a)

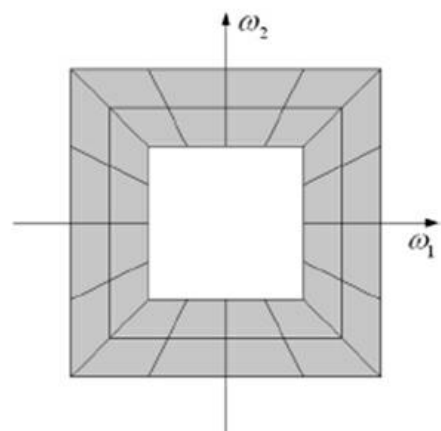

(b)

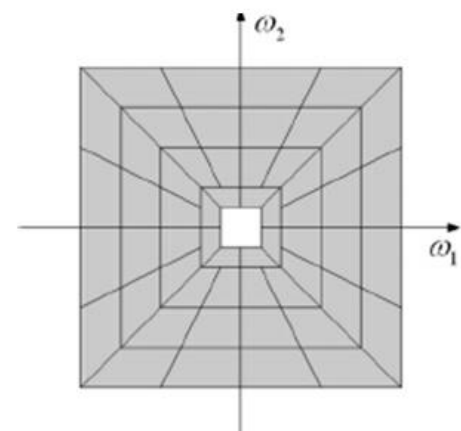

(c)

Figure 2. Frequency Resolution of (a) Contourlet, (b) MDFB and (c) FMDFB

\subsection{Laplacian Pyramid}

It is an over complete decomposition in which input image is represented by a set of band pass images and a low pass image. The theory behind the construction of Laplacian Pyramid is borrowed from Gaussian pyramid. Gaussian pyramid of an image is constructed by successively lowpass filtering with the Gaussian kernel and down sampling the low pass filtered image. As a result, an image is represented with a set of low pass images G0, G1, G2, ... Gn at different resolutions as shown in Figure 3. The relationship between Gaussian and Laplacian pyramids is shown in Figure 4. As seen from Figure 4, the Gaussian pyramid shows high degree of correlation among the lowpass images $G_{0}, G_{1}, G_{2}, \ldots, G_{n}$. This is not a desirable characteristic of a compact image representation [8].

The Laplacian pyramid eliminates this problem by representing the image as a set of band pass images and a lowpass image. The image $\left(\mathrm{G}_{0}\right)$ is first lowpass filtered to get the reduced (decimated/down sampled) version $G_{1}$ called as coarse approximation as in the case of Gaussian pyramid. Then, $\mathrm{G}_{1}$ is expanded (interpolated / upsampled) to get $\mathrm{E}_{1}$, and the prediction $L_{1}$ is obtained by subtracting $E_{1}$ from $G_{1}$. This process can be iterated on the lowpass image up to the deserted level of decomposition to generate other bandpass images in lower frequency range. At each level of decomposition, the Laplacian prediction is computed as $L_{n}=G_{n}-E_{n}$. These $L_{n}$ are the set of bandpass images that constitutes the Laplacian pyramid. It is worth mentioning that, at each level of decomposition, the image resolution is reduced by a factor 4 . The major drawback of Laplacian pyramid is the implicit oversampling.

A block diagram of single stage Laplacian pyramid decomposition is shown in Figure 5. An interesting feature of the Laplacian pyramid is that decimation is performed only on the 
lowpass image. Thus, there is no frequency scrambling in Laplacian pyramid unlike separable wavelets. Further, directional decomposition is performed on the bandpass images at each scale [4]. Also, the Laplacian pyramid reconstruction is straight forward as shown in Figure 5.

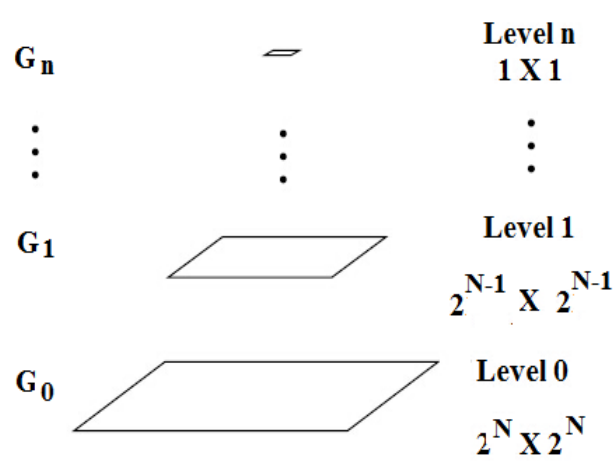

(a)

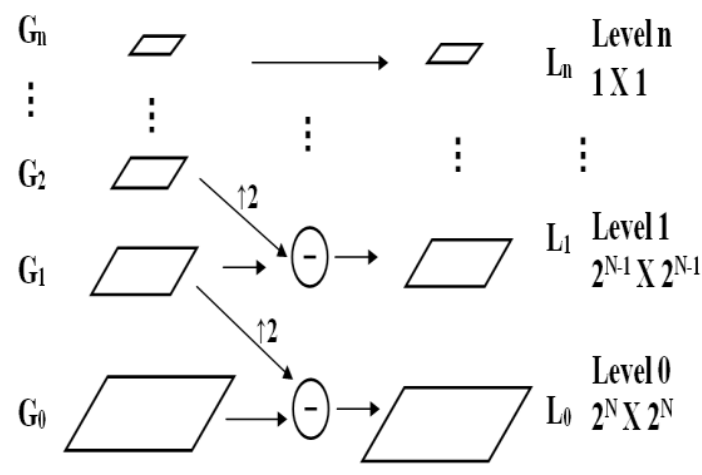

(b)

Figure 3. (a) Structure of Gaussian Pyramid (b) Structure of Laplacian Pyramid

Gaussian
Pyramid

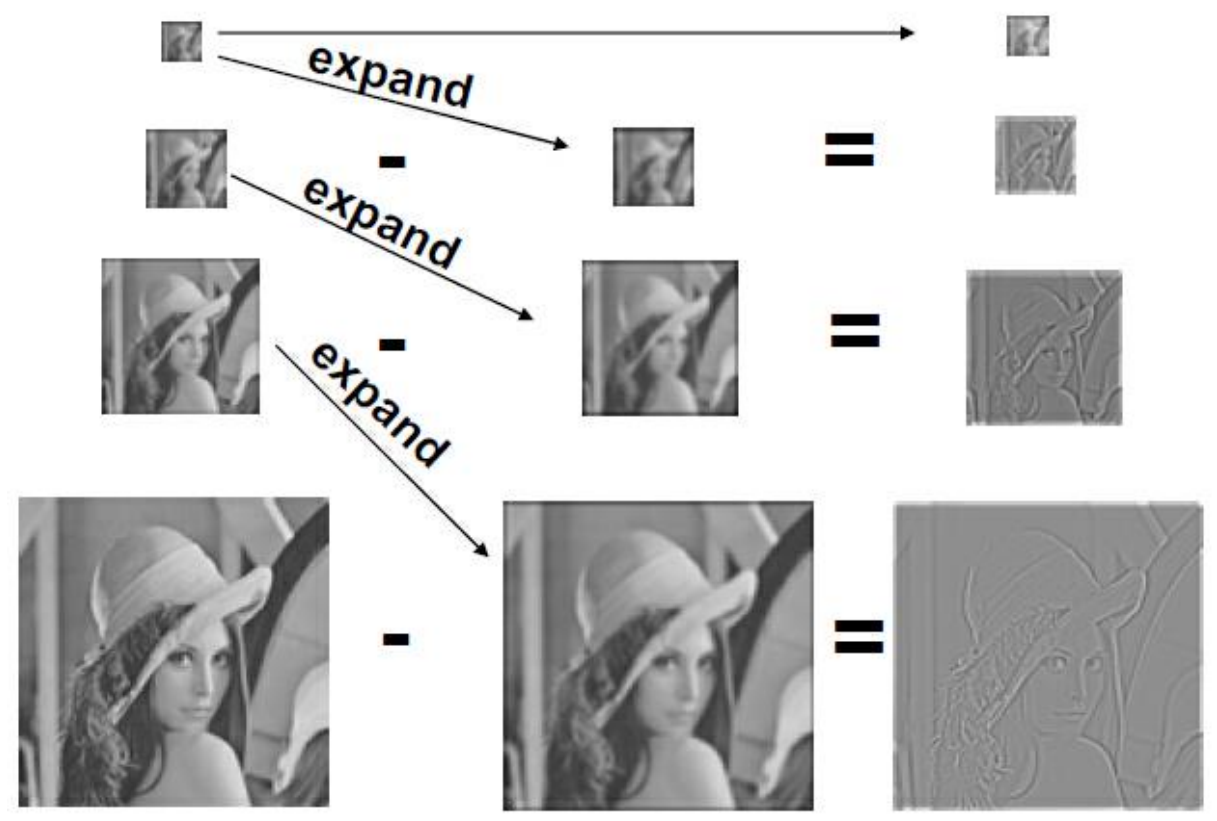

Figure 4. Relationship between Gaussian and Laplacian Pyramids

To avoid aliasing in the LP, it requires the stopband edge of $h_{L}[n]$ should be less than $\Pi / 2$. In order to satisfy the criteria, equiripple $\mathrm{hL}[\mathrm{n}]$ with pass band edge $0.3 \Pi$ and stop band edge $0.5 \Pi$ are designed $[9,10]$. Also, the Laplacian pyramid is designed with orthogonal filters i.e., $\mathrm{h}_{\mathrm{L}}[\mathrm{n}]=\mathrm{g}_{\mathrm{L}}[-\mathrm{n}]$ to ensure lossless reconstruction. 


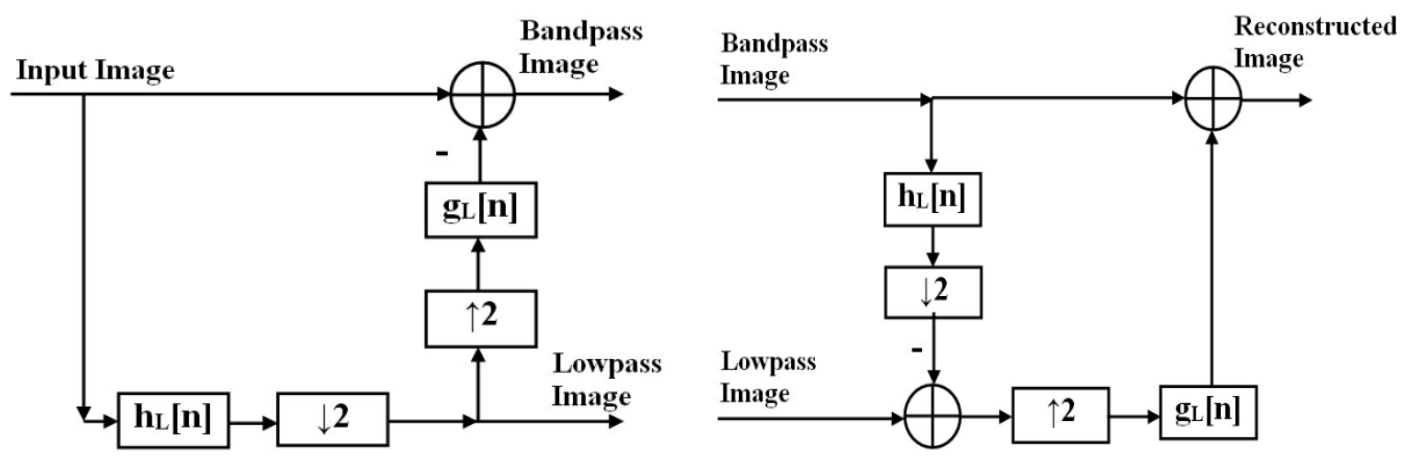

Figure 5. Single Stage Analysis and Synthesis Structure of the Laplacian Pyramid $h_{L}[n]$ and $g_{L}[n]$ are the Lowpass Analysis and Synthesis Filters. $\downarrow$ 2 and $\uparrow 2$ Represents Down Sampling and Upsampling by a Factor of 2 Respectively

\subsection{Directional Filter Bank}

Directional filter bank (DFB) was introduced by Bamberger and Smith in 1992 [11]. It is a 2-D directional filter bank that performs directional decomposition with perfect reconstruction and maximally decimated property. The DFB partitions a frequency plane into a set of wedge-shape region as illustrated in Figure 6. It can be implemented efficiently in an ' $l$ ' level tree structure that results in $2^{l}$ directional subbands. The tree structure is implemented with a two-channel filter bank in which a complementary diamond-shaped filter pair is followed by a quincunx down sampling as shown in Figure 7 [2].

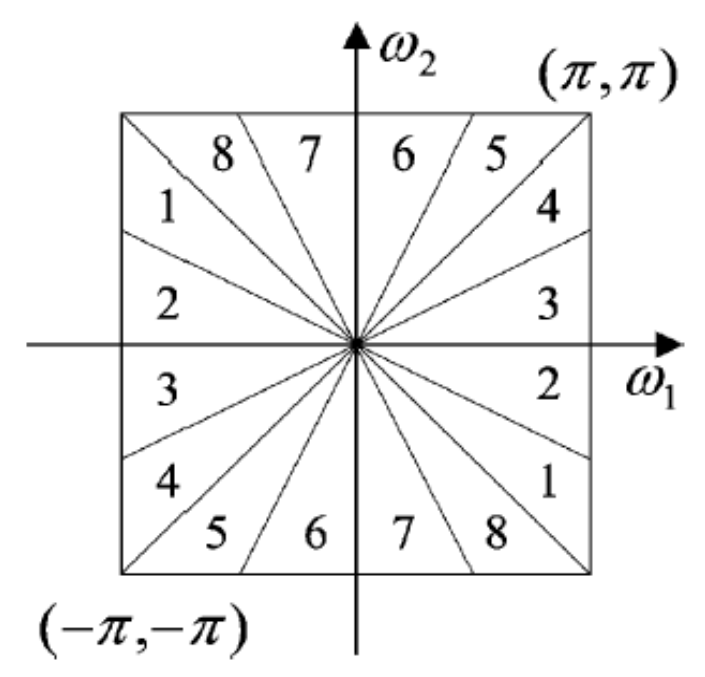

Figure 6. Frequency Plane Partitioning in a Three-level DFB 


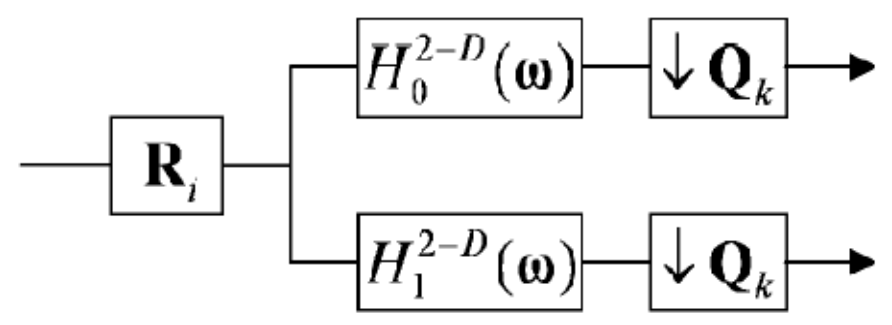

\section{Figure 7. Two Channel DFB( Ri - Resampling Matrix,H0,H1 - Low Pass \\ Filters, Qk - Quincunx Down Sampling)}

A resampler is employed before the two-channel filter bank. Its function is to shear the desired frequency partitions into diamond shape so that the two-channel filter bank can give the desired frequency bands. There are many resampling matrices have been proposed in the literature [2]. Any one of the following can be used according to the required frequency band.

$$
R_{1}=\left(\begin{array}{ll}
1 & 1 \\
0 & 1
\end{array}\right) \quad R_{2}=\left(\begin{array}{cc}
1 & -1 \\
0 & 1
\end{array}\right) \quad R_{3}=\left(\begin{array}{ll}
1 & 0 \\
1 & 1
\end{array}\right) \quad R_{4}=\left(\begin{array}{cc}
1 & 0 \\
-1 & 1
\end{array}\right)
$$

The lowpass filters $\mathrm{H}_{0}, \mathrm{H}_{1}$ can be derived from a diamond shaped filter pair that splits the frequency spectrum of the input signal into lowpass and highpass channels [12]. Figure 8 shows the frequency spectrum of diamond shaped filter.

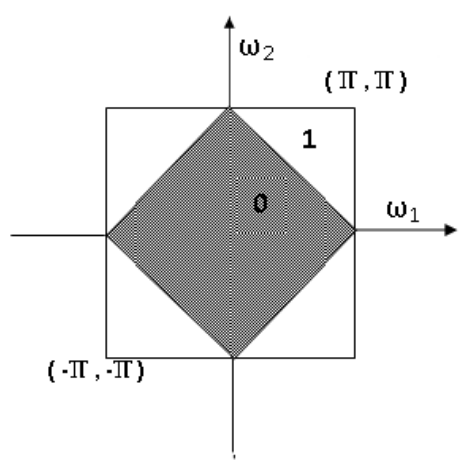

Figure 8. Frequency Spectrum of Diamond Shaped Filter (Stopband is shown in Gray) 


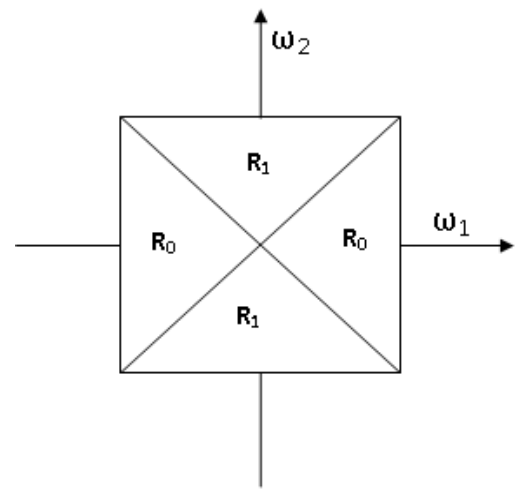

Figure 9. Spectral Regions R0 and R1

One filter pair can be derived from the other by simply modulating the filters by $\Pi$ in either the $\omega_{1}$ or $\omega_{2}$ frequency variable. Perfect reconstruction is achieved by applying the same modulation to both analysis and synthesis filters. Because of sampling, subbands would suffer from spatial distortion. Spatial distortion results from resampling used in the construction. This problem can be solved by adopting backsampling at the output of the DFB $[2,6]$. The overall sampling matrix is given by

$$
S=\left\{\begin{array}{lll}
D_{0}^{i-2} & \text { in } & R_{0} \\
D_{1}^{i-2} & \text { in } & R_{1}
\end{array}\right.
$$

where ' $i$ ' is the index of output stage of DFB, $R_{j}, j=0,1$ are the spectral regions shown in Figure 9, D0 and D1 are down sampling matrices.

$$
D_{0}=\left(\begin{array}{ll}
2 & 0 \\
0 & 1
\end{array}\right) \quad D_{1}=\left(\begin{array}{ll}
1 & 0 \\
0 & 2
\end{array}\right)
$$

The backsampling reorders the subbands so that the overall sampling is diagonal [13][14].

The quincunx down sampling matrices generates the same sub-lattice but the down sampling operation rotates the input image by $-45^{\circ}$ and $45^{\circ}$. The sampling matrices that can be used in directional filter banks are derived as follows.

$$
\begin{aligned}
& Q_{1}=R_{2} D_{0} R_{3}=R_{3} D_{1} R_{2} \\
& Q_{2}=R_{1} D_{0} R_{4}=R_{4} D_{1} R_{1}
\end{aligned}
$$

\section{Design of FMDFB Filters}

The design of FMDFB structure requires FIR filters that are separately designed for Laplacian pyramid, directional filter bank and higher scale decomposition stages as described below.

\subsection{Design of Laplacian Pyramid Filters}

The low-pass analysis filter $\mathrm{hL}[\mathrm{n}]$ and low-pass synthesis filter $\mathrm{g}_{\mathrm{L}}[\mathrm{n}]$ of the Laplacian pyramid shown in Figure 3.6 are derived assuming that $h_{L}[n]$ and $g_{L}[n]$ are time reversal. Moreover, these filters are realized using separable 1D filters. Any 1D low-pass filters that belong to the wavelet family can be used to derive $h_{L}[n]$. We have used the well known ' $5 / 3$ ', 
'9/7'[15], pkva [16], and Burt filter [17] as $h_{L}[n]$. These low-pass filters are designed to have cutoff frequency around $\Pi / 2$.

\subsection{Design of Directional Filter Bank Filters}

FMDFB uses DFB similar to the ones proposed by Bamberger and Smith. DFB is constructed with a tree structured two channel filter bank that is formed with complementary fan-shaped filters followed by quincunx downsampling. The fan-shaped filters are obtained from complementary diamond shaped filters by simple modulation. This filter bank is designed to achieve perfect reconstruction by using the same modulation for analysis and synthesis filters [18]. We use the filters proposed by Phoong, et al., of support sizes $23 \times 23$ and $45 \times 45$.

\subsection{Smoothening Filters}

The smoothing filters are the ones denoted $a_{s} h_{C}[n]$ in Figure 1. This filter offers scale decompositions at higher scales. The function of this $\mathrm{h}_{C}[\mathrm{n}]$ is similar to the Laplacian pyramid filter $h_{L}[n]$. So, the same filter coefficients used in Laplacian pyramid can be used as $h_{C}[n]$. However, to control the computational complexity in higher scales, a simple binomial filter of length 7 termed as binom7 is used. Also, the binom 7 filter is alias-free in nature [18].

\section{Experimental Results and Discussion}

The filters discussed in previous section are designed using MATLAB filter design toolbox for the given specifications. Figure 10 shows the plot of normalized frequency Vs normalized magnitude of low-pass analysis and synthesis filters of type 'pkva'.

Figure 11 shows the magnitude responses of diamond-shaped filter pair of support sizes 23 $\times 23$ and $45 \times 45$ respectively. The magnitude responses of complementary fan filters of support size $23 \times 23$ and $45 \times 45$ [16] are shown in Figure 12 and Figure 13 respectively. The conventional wavelet filters such as Haar, 5/3, and 9/7 can also be used to derive 2D diamond and fan shaped filter pairs. The designed FIR filter coefficients are listed in Table I with their respective lengths.
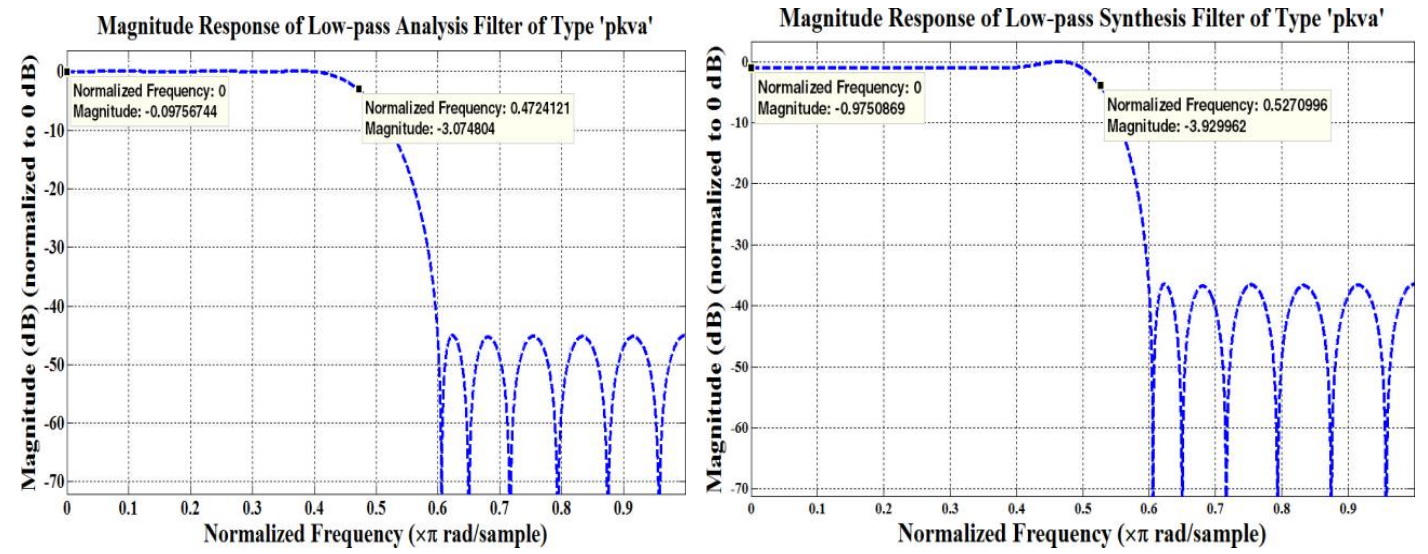

Figure 10. Frequency Response of Low-pass Analysis $\left(h_{L}[n]\right)$ and Synthesis Filters ( $\left.g_{\mathrm{L}}[\mathrm{n}]\right)$ of Type 'pkva' with Cutoff Frequency Around $\Pi / 2$ 

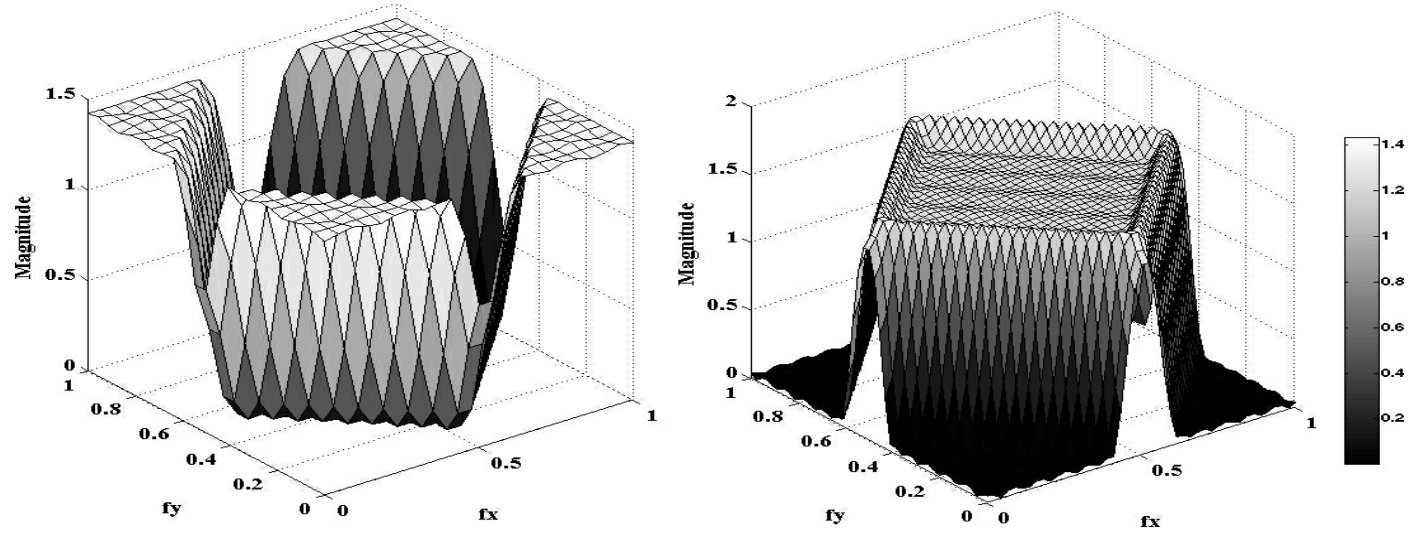

Figure 11. Magnitude Responses of Diamond-shaped Filter Pair of Support sizes $23 \times 23$ and $45 \times 45$ Respectively
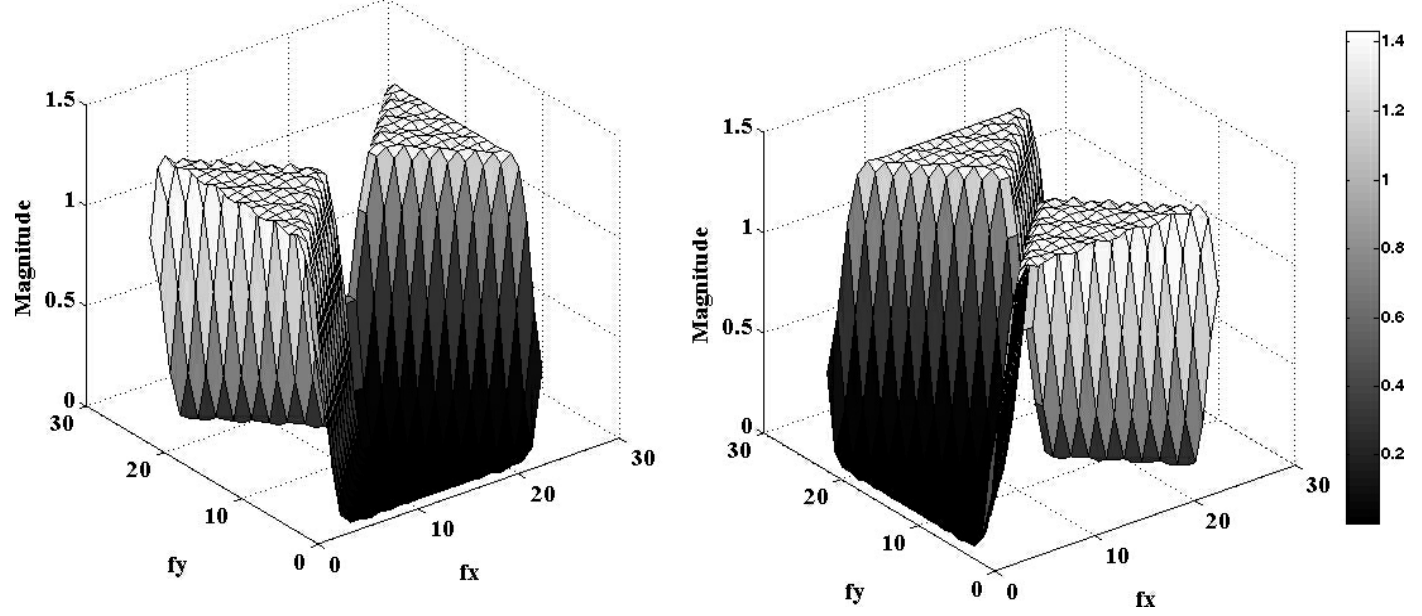

Figure 12. Magnitude Responses of Complementary Fan-shaped Filters of Support Sizes $23 \times 23$
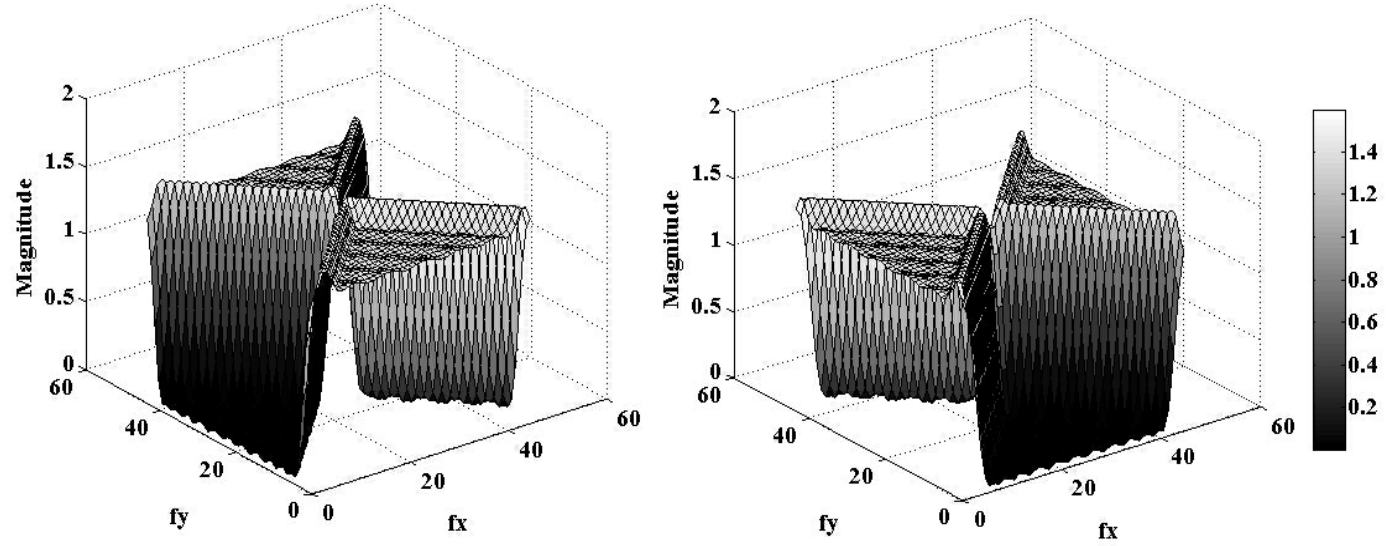

Figure 13. Magnitude Responses of Complementary Fan-shaped Filters of Support Sizes $45 \times 45$ 
Table 1. Designed Filter Coefficients for FMDFB Analysis and Synthesis Structures

\begin{tabular}{|c|c|c|c|c|}
\hline Filter Name & Analysis Filter $(\mathrm{H})$ & Length & Synthesis Filter(G) & Length \\
\hline Burt & $\begin{array}{l}-0.070710678,0.353553, \\
0.848528,0.353553 \\
0.07071\end{array}$ & 5 & $\begin{array}{l}-0.01515,-0.07576,0.368706, \\
0.85863,0.368706,-0.07576,- \\
0.01515\end{array}$ & 7 \\
\hline $5 / 3$ & $\begin{array}{l}-0.176776695,0.353553, \\
1.06066,0.353553,- \\
0.17678\end{array}$ & 5 & $0.353553,0.707107,0.353553$ & 3 \\
\hline $9 / 7$ & $\begin{array}{l}0.037828456, \\
0.023849465, \\
0.110624404, \\
0.377402856, \\
0.852698679,0.377402856 \\
,-0.110624404,- \\
023849465,0.03782845\end{array}$ & 9 & $\begin{array}{l}-0.064538883, \\
0.040689418,0.418092273, \\
0.788485616,0.418092273,- \\
0.040689418,-0.064538883\end{array}$ & 7 \\
\hline pkva & $\begin{array}{ll}-0.010182338,0, & \\
0.019233304,0, & \\
0.037193817,0, & \\
0.068730779,0, & - \\
0.136471609,0, & \\
0.445477272, & \\
0.707106781, & \\
0.445477272,0, & \\
0.136471609, & - \\
0.0 .068730779,0, & - \\
0.037193817,0, & - \\
0.019233304,0, & - \\
0.010182338, & -\end{array}$ & 23 & $\begin{array}{l}-0.000146626,0,0.000553919, \\
0,-0.001594328,0, \\
0.00400279,0,-0.009625731, \\
0,0.027484279, \\
-0.010182338,-0.032441663, \\
0.019233304,0.045229944, \\
-0.037193817,-0.056672091, \\
0.068730779,0.066186496,- \\
0.136471609,-0.072358633, \\
0.445477272,0.781620562, \\
0.445477272,-0.072358633,- \\
0.136471609,0.066186496, \\
0.068730779,-0.056672091,- \\
0.037193817,0.045229944, \\
0.019233304,-0.032441663,- \\
0.010182338,0.027484279,0,- \\
0.009625731,0,0.00400279, \\
0, \quad-0.001594328, \\
0,0.000553919,0, \\
-0.000146626 \quad\end{array}$ & 45 \\
\hline Binom7 & $\begin{array}{l}0.015625,0.09375, \\
0.234375,0.3125, \\
0.234375,0.09375 \\
0.015625\end{array}$ & 7 & $\begin{array}{l}0.015625,0.09375,0.234375 \\
0.3125,0.234375,0.09375 \\
0.015625\end{array}$ & 7 \\
\hline
\end{tabular}

\subsection{Discussion}

In the design of FMDFB, we use different filters such as pkva, Burt, Haar, 5/3, and 9/7 in both analysis and synthesis structures. The magnitude responses of these filters are compared in Figure 14. The 'pkva' is an equiripple filter that has maximum ripple magnitude of about - 
$45 \mathrm{~dB}$ with 23 coefficients. The FMDFB structure is tested with various filter combinations of pyramidal and directional filters. As evident from Table II, the FMDFB exhibits perfect reconstruction irrespective of the filters used showing zero mean square error (MSE), infinite peak signal to noise ratio (PSNR), and structural and feature similarity indices of unity. The computation time depends on the length of the FIR filters and the number of pixels processed. However, the 'pkva' filter is preferred for pyramidal and directional decomposition because of its alias-free nature as it shows less magnitude above the frequency $\Pi / 2$. Yet, the binomial filter of length 7 that shows drastic fall in magnitude beyond $\Pi / 2$ (Figure 14) is used for pyramidal decomposition at higher scales so as to reduce computational overhead.

Table 2. Quantitative Results Showing Perfect Reconstruction of FMDFB and Computation Time of Barbara Image

\begin{tabular}{|l|c|c|c|c|c|c|c|}
\hline $\begin{array}{c}\text { Directional } \\
\text { Filter }\end{array}$ & $\begin{array}{c}\text { Pyramidal } \\
\text { Filter }\end{array}$ & MSE & PSNR & SSIM & FSIM & \multicolumn{2}{|c|}{$\begin{array}{c}\text { Time elapsed } \\
\text { in seconds }\end{array}$} \\
\cline { 6 - 8 } & & & & & $\mathbf{2 5 6 \times 2 5 6}$ & $\mathbf{5 1 2 \times 5 1 2}$ \\
\hline $5 / 3$ & burt & 0 & Inf & 1 & 1 & 0.218 & 0.4619 \\
\hline $5 / 3$ & $5 / 3$ & 0 & Inf & 1 & 1 & 0.2264 & 0.4497 \\
\hline $5 / 3$ & $9 / 7$ & 0 & Inf & 1 & 1 & 0.2207 & 0.4925 \\
\hline $5 / 3$ & pkva & 0 & Inf & 1 & 1 & 0.2596 & 0.5633 \\
\hline $9 / 7$ & burt & 0 & Inf & 1 & 1 & 0.2424 & 0.5071 \\
\hline $9 / 7$ & $5 / 3$ & 0 & Inf & 1 & 1 & 0.2364 & 0.5002 \\
\hline $9 / 7$ & $9 / 7$ & 0 & Inf & 1 & 1 & 0.2385 & 0.5294 \\
\hline $9 / 7$ & pkva & 0 & Inf & 1 & 1 & 0.2627 & 0.6184 \\
\hline pkva & burt & 0 & Inf & 1 & 1 & 0.6293 & 1.289 \\
\hline pkva & $5 / 3$ & 0 & Inf & 1 & 1 & 0.4941 & 1.2082 \\
\hline pkva & $9 / 7$ & 0 & Inf & 1 & 1 & 0.5019 & 1.2591 \\
\hline pkva & pkva & 0 & Inf & 1 & 1 & 0.5423 & 1.2984 \\
\hline Haar & burt & 0 & Inf & 1 & 1 & 0.1933 & 0.4135 \\
\hline Haar & $5 / 3$ & 0 & Inf & 1 & 1 & 0.1825 & 0.4154 \\
\hline Haar & $9 / 7$ & 0 & Inf & 1 & 1 & 0.1995 & 0.453 \\
\hline Haar & pkva & 0 & Inf & 1 & 1 & 0.2145 & 0.5127 \\
\hline
\end{tabular}




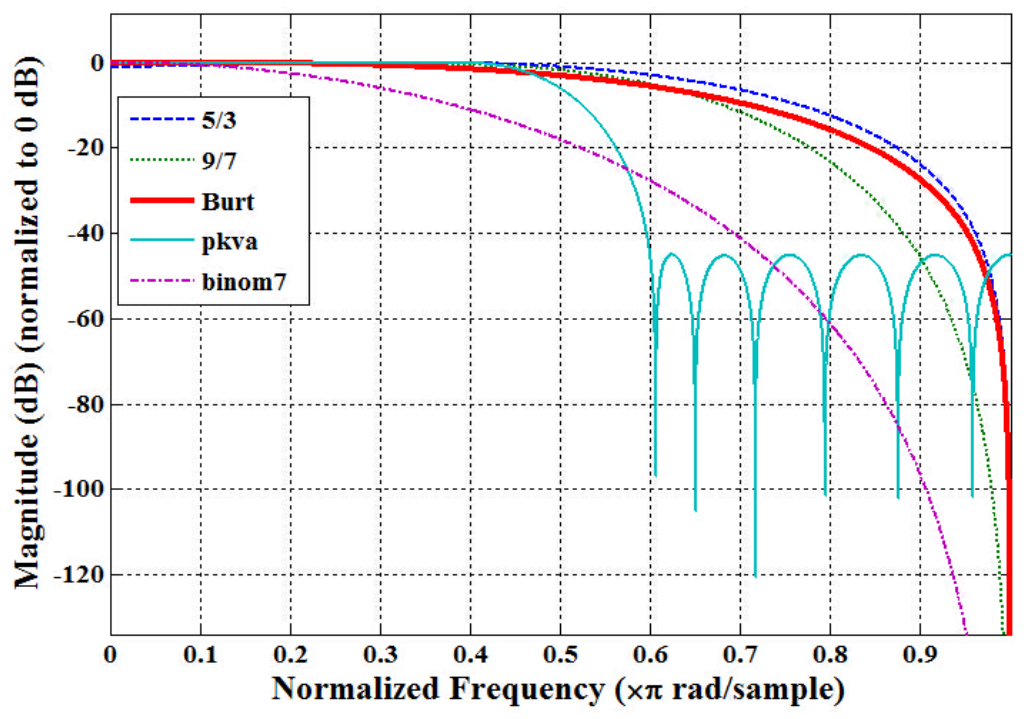

Figure 14. Comparison of the Magnitude Responses of Filters used for Pyramidal and Directional Decomposition

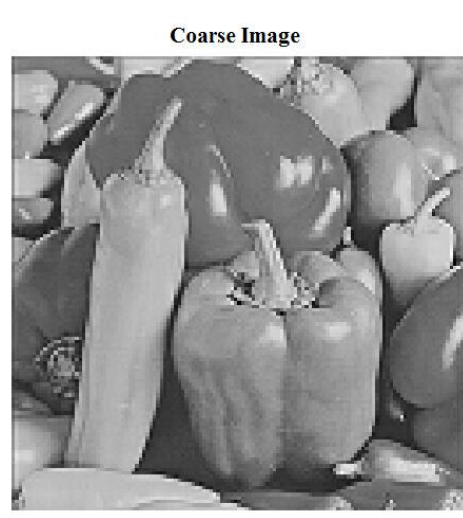

(a)

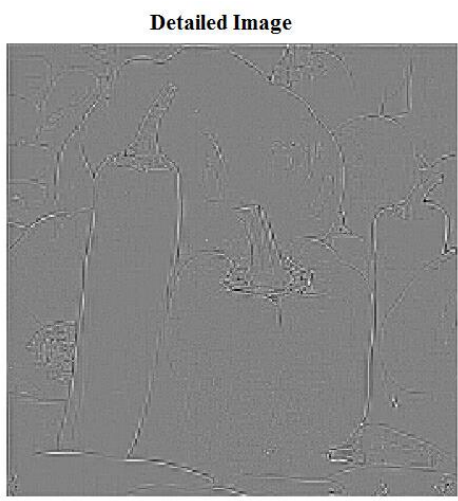

(b)

First Level DFB Subbands

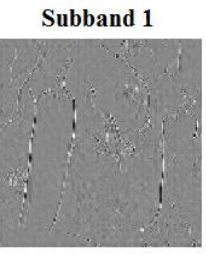

Subband 3

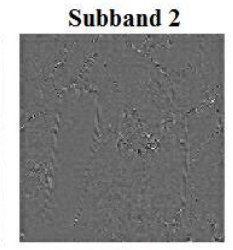

Subband 4

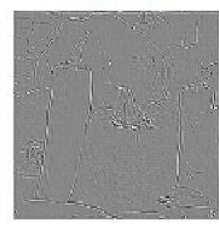

(c)
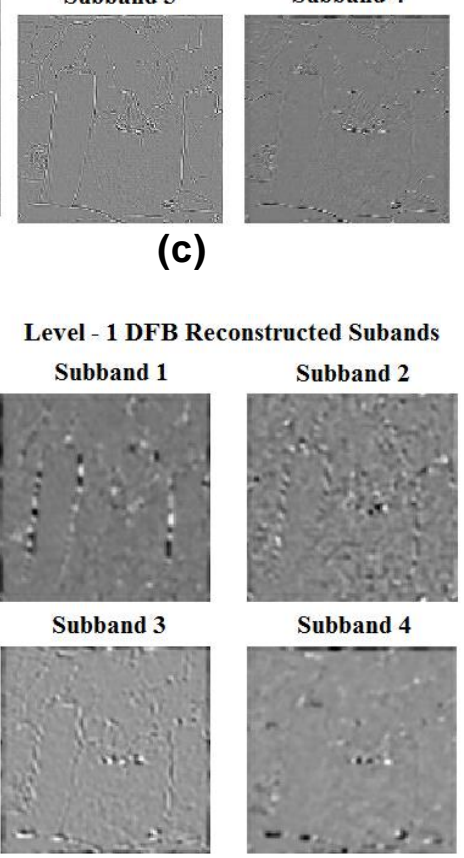

(f)

(d) (e)

Second Level DFB Subbands



Smoothened Subband 3

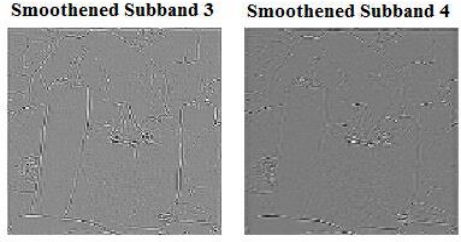

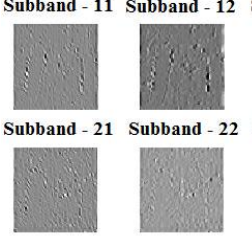

Subband - 31 Subband - 32 Subband - 33 Subband - 34

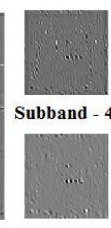



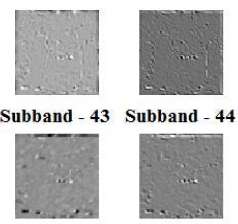

and - 13 Subband - 14
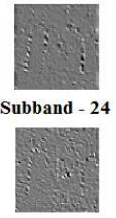


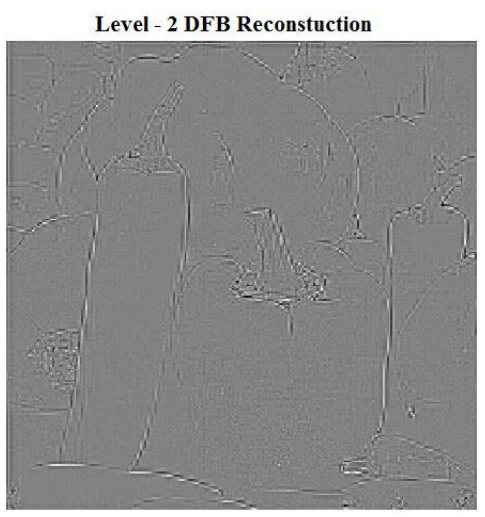

(g)

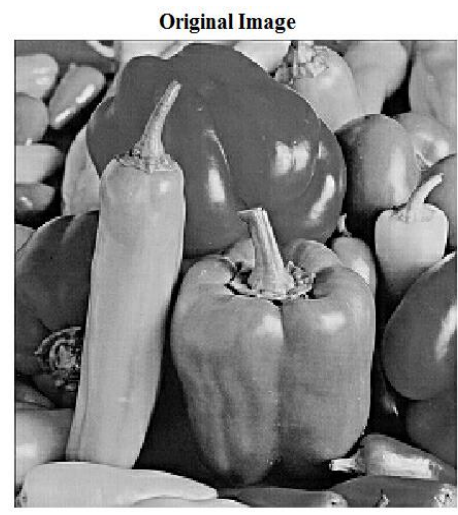

(h)

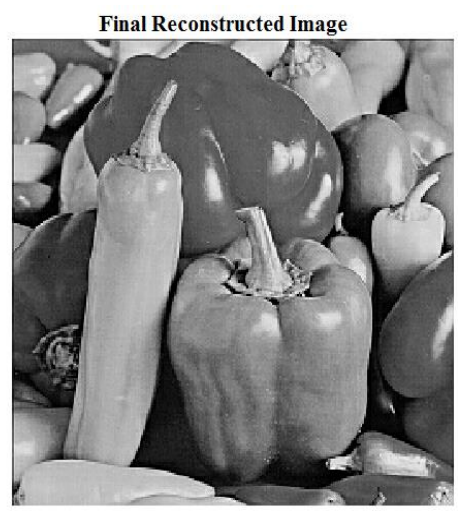

(i)

Figure 15. Experimental Images Showing the Perfect Reconstruction of FMDFB (a) Coarse Image and (b) Detailed Image Obtained from Laplacian Pyramid (c) First level DFB Subbands (d) Smoothened First Level Subbands (e) Second Level DFB Subbands (f) First Level DFB Reconstruction (g) Second Level DFB Reconstruction (h) Original Image (i) Final Reconstructed Image

The resultant images at various stages of FMDFB are shown in Figure 15. The number of scale (s) and directional (l) decomposition is restricted to 2 [19].The original image of size $256 \times 256$ is fed as input to the FMDFB. At the first scale decomposition, the image is decomposed into coarse (low-pass) and detailed (band-pass) images of size $128 \times 128$ and $256 \times 256$ respectively. Then, a two $(1=2)$ level DFB is applied on the band-pass (detailed) image to decompose it into $2^{l}\left(2^{2}=4\right)$ subbands each of size $128 \times 128$. Again, second level scale decomposition is performed on each of the four subbands and the resultant subbands are termed as smoothened subbands. Then, second level DFB is applied on each of the smoothened subband. This result in sixteen $\left(2^{l} \times 2^{s}=2^{2} \times 2^{2}\right)$ subbands each of size $64 \times 64$, four from each smoothened subband as the number of DFB levels is chosen as 2. The FMDFB reconstruction follows the straight forward procedure. All the sixteen subbands are given as input to the synthesis filter. The first level DFB reconstruction results in 4 subbands of size $128 \times 128$, the second level DFB reconstruction followed by Laplacian reconstruction result in an image of size $256 \times 256$ ), that is same as that of the original image.

\section{Conclusion}

This paper presented the FIR filter design procedure required to construct the fast multiscale directional filter banks. The design of three different types of FIR filters is discussed. Also, the perfect reconstruction with the designed FIR filters and critical sampling property of FMDFB is demonstrated with the help of stage by stage output images. This FMDFB structure is useful for several image processing applications such as feature extraction, image retrieval, and image denoising.

\section{References}

[1] S. Sutha, E. J. Leavline and D. Singh, WSEAS Trans. Signal Process, vol. 9, (2013).

[2] M. N. Do and M. Vetterli, IEEE Trans. on Image Process, vol. 14, no. 12, (2005).

[3] L. E.Jebamalar, S. Sutha and D. Singh, Int. J. Comput. Appl., vol. 33, no. 10, (2011).

[4] M. N. Do and M. Vetterli, IEEE Trans. on Image Process, vol. 14, no. 12, (2005). 
[5] K. O. Cheng, N. F. Law and W. C. Siu, Pattern Recognit., vol. 40, no. 4, (2007).

[6] K. O. Cheng, N. F. Law and W. C Siu, IEEE Trans. on Image Process, vol. 16, no. 8, (2007).

[7] E. J. Leavline, S. Sutha and D. A. A. G. Singh, Int. J. Innov. Comput. Inf. Control, vol. 10, no. 3, (2014).

[8] P. J. Burt and E. H. Adelson, IEEE Trans. Commun., vol. 31, no. 4, (1983).

[9] E. J. Leavline, S. Sutha and D. A. A. G. Singh JOSA A, vol. 31, no. 2, (2014).

[10] M. N. Do and M. Vetterli, IEEE Trans. On Signal Process, vol. 51, no. 9, (2003).

[11] S. M. Phoong, C. W. Kim, P. P. Vaidyanathan and R. Ansari, IEEE Trans. Signal Process, vol. 43, no. 3, (1995).

[12] M. N. Do, "Directional multi resolution image representations", Citeseer, vol. 2500, (2001).

[13] K. O. Cheng, N. F. Law and W. C. Siu, IEEE Trans. on Image Process, vol. 16, no. 8, (2007).

[14] S. Sutha, E. Jebamalar Leavline and D. A. A. G. Singh, Inf. Technol. J., vol. 12, no. 7, (2013).

[15] K. P. Soman, "Insight into wavelets: From theory to practice", PHI Learning Pvt. Ltd., (2010).

[16] S.M. Phoong, C. W. Kim, P. P. Vaidyanathan, and R. Ansari, IEEE Trans. on Signal Process., 43, 3 (1995).

[17] P. J. Burt and E. H. Adelson, IEEE Trans. on Commun., vol. 31, no. 4, (1983).

[18] K. O. Cheng, N. F. Law and W. C. Siu, IEEE Trans. on Image Process, vol. 16, no. 8, (2007).

[19] E. J. Leavline and S. Sutha, Proceedings of the International Conference on Recent Trends in Information Technology, (2011).

\section{Authors}

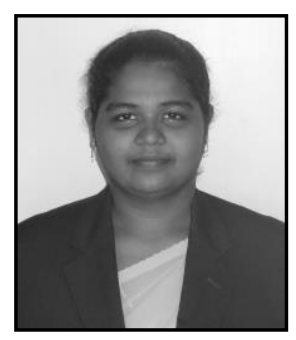

E. Jebamalar Leavline, she is with Department of Electronics and Communication Engineering, Bharathidasan Institute of Technology, Anna University, Tiruchirappalli, Tamil Nadu, India. She received her B.E, Electronics and Communication Engineering in 2005 and M.E in Applies Electronics in 2008 from Anna University, Chennai, Tamil Nadu, India. Also she is a M.B.A graduate in Education Management. She has published a good number of research papers in reputed International Journals and conferences. Her research interests include image processing, signal processing, VLSI design, data mining, teaching learning process and engineering education. She is a Review committee member of several reputed journals and also she is a member of ISTE and IAENG.

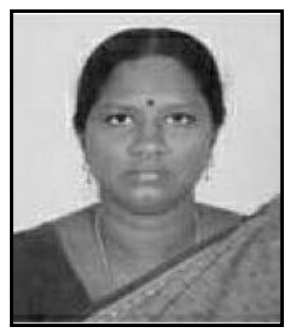

S. Sutha, she received B. E. from Government College of Engineering, Tirunelveli, Manonmaniam Sundaranar University in 1996, M. E. from College of Engineering, Guindy, Anna University Chennai in 2000, and Ph.D from Anna University Chennai in 2008. Presently she is working as Assistant Professor (SG) in University College of Engineering, Dindigul, Tamil Nadu, India. Her research interests include power system, image processing, and VLSI design. 